\title{
Les récepteurs olfactifs et le codage neuronal de l'odeur
}

Le système olfactif se distingue des autres systèmes sensoriels par de nombreux aspects, et avant tout par l'existence d'un très grand nombre de gènes codant pour des récepteurs olfactifs aux propriétés distinctes. En dépit de cette multiplicité des récepteurs, et quoique certaines anosmies (c'est-à-dire pertes de l'odorat) soient sélectives, il est hautement probable qu'il n'existe pas un récepteur particulier par odeur. En fait, les cellules réceptrices sont, individuellement, sensibles à des molécules de structure chimique et d'odeur très différentes et, dans les bulbes olfactifs, plusieurs centaines d'entre elles convergent sur les cellules mitrales. La spécificité de la reconnaissance des odeurs semble mettre en jeu plusieurs types de mécanismes. Certains récepteurs reconnaissent très spécifiquement un petit nombre de ligands mais, le plus souvent, une entité moléculaire donnée, et donc une odeur, semble codée par une combinatoire plurineuronale de topologie particulière, aboutissant à une "chimiotopie" se manifestant au niveau

André Holley Gilles Sicard des cellules sensorielles de l'épithélium aussi bien qu'au niveau du bulbe olfactif. C'est à ce dernier niveau que les glomérules olfactifs intègrent les afférences sensorielles et projettent vers les aires paléocorticales.

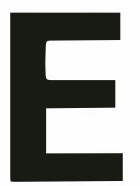

n révélant l'appartenance des récepteurs olfactifs à une superfamille de récepteurs à sept domaines transmembranaires, liés à une protéine $G$, les recherches récentes en biologie moléculaire [13] ont rapproché le champ, jusqu'alors isolé, de la chimioréception olfactive des autres secteurs de la communication cellulaire $(\mathrm{m} / \mathrm{s}$, $n^{\circ} 6$ vol. 7, p. 616). Mais en annonçant la grande diversité des séquences codant pour les protéines réceptrices, les mêmes travaux confirmaient la singularité du système olfactif au sein des systèmes sensoriels. En effet, si les récepteurs différents se chiffrent par centaines, comme on le pense désormais, le sens olfactif fait montre d'une stratégie vraiment originale pour capter et différencier ses stimuli spécifiques. Une autre source de complexité est fournie par la pluralité des voies de transduction puisque les récepteurs peuvent contrôler plusieurs messagers intracellulaires, AMPc et IP3 [4]. 


\section{Une stratégie originale pour la réception}

En combinant les réponses au stimulus lumineux unidimensionnel de trois types seulement de photorécepteurs, la vision construit les nuances subtiles des couleurs. Il est vrai que pour pouvoir coder l'agencement spatial des figures colorées, la rétine reproduit de multiples fois le motif de base à trois récepteurs, de sorte que la dimension spatiale du stimulus est conservée dans l'organisation du message qui atteint les aires visuelles. En ce qui concerne l'audition, la cochlée réalise sur le stimulus acoustique, également unidimensionnel, une analyse physique que l'on peut qualifier de spatialisation puisque les fréquences sonores se distribuent régulièrement sur la lame sensorielle. C'est donc la position des récepteurs sur la cochlée et non leurs propriétés détectrices singulières, qui assume, pour l'essentiel, le codage du stimulus. Quant à la somesthésie, elle utilise bien plusieurs sortes de récepteurs dans ses différentes modalités, mais c'est encore la disposition des récepteurs à la surface du corps, donc leur organisation spatiale, qui prend en charge la plus grande partie de l'information.

Avec ses très nombreux récepteurs, le système olf actif paraît donc suivre un tout autre principe de construction. La raison de cette singularité doit sans doute être recherchée dans la nature des stimuli. Chaque espèce chimique assez volatile pour atteindre l'épithélium olfactif à concentration supraliminaire se voit affecter une qualité perçue qui lui est propre, son odeur. Cette identification n'est réalisable qu'à l'intersection d'un grand nombre de propriétés moléculaires. Poids moléculaire, densité électronique, polarisabilité, présence de tel ou tel groupe fonctionnel... au-

\section{RÉFÉRENCES}

1. Buck LB, Axel R. A novel multigene family may encode odorant receptors : a molecular basis for odor recognition. Cell $1991 ; 65: 175-87$.

2. Nef $P$, Heinemann S, Dionne VE. Spatial distribution of OR3, a putative seven-transmembrane domain olfactory receptor, reveals an olfactory map. Chem Senses 1991 ; $16: 562$.

3. Parmentier M, Libert F, Schurmans S, et al. Members of the putative olfactory receptor gene family are expressed in mammalian germ cells. Nature $1992 ; 355: 453-5$.

4. Breer H, Boekhoff I, Tareilus E. Rapid kinetics of second messenger formation in olfactory transduction. Nature $1990 ; 345 ; 65-8$.

5. Revial MF, Duchamp A, Holley A. Odour discrimination by frog olfactory receptors : a second study. Chem Senses 1978; 3:7-21.

6. Sicard G, Holley A. Receptor cell responses to odorants : similarities and differences among odorants. Brain Res 1984 . 292: 283-96.

7. Raning K, Krieger J, Strotmann J, Boekhoff I, Kubick S, Baumstark C, Breer $\mathrm{H}$. Cloning and expression of odorant receptors. Nature 1993 ; 362: 353-6.

8. Lancet D, Ben-Arie N. Olfactory receptors. Curr Biol $1993: 3: 668-74$

9. Ressler KJ, Sullivan SS, Buck LB. A zonal organization of odorant receptor gene expression in the olfactory epithelium. Cell

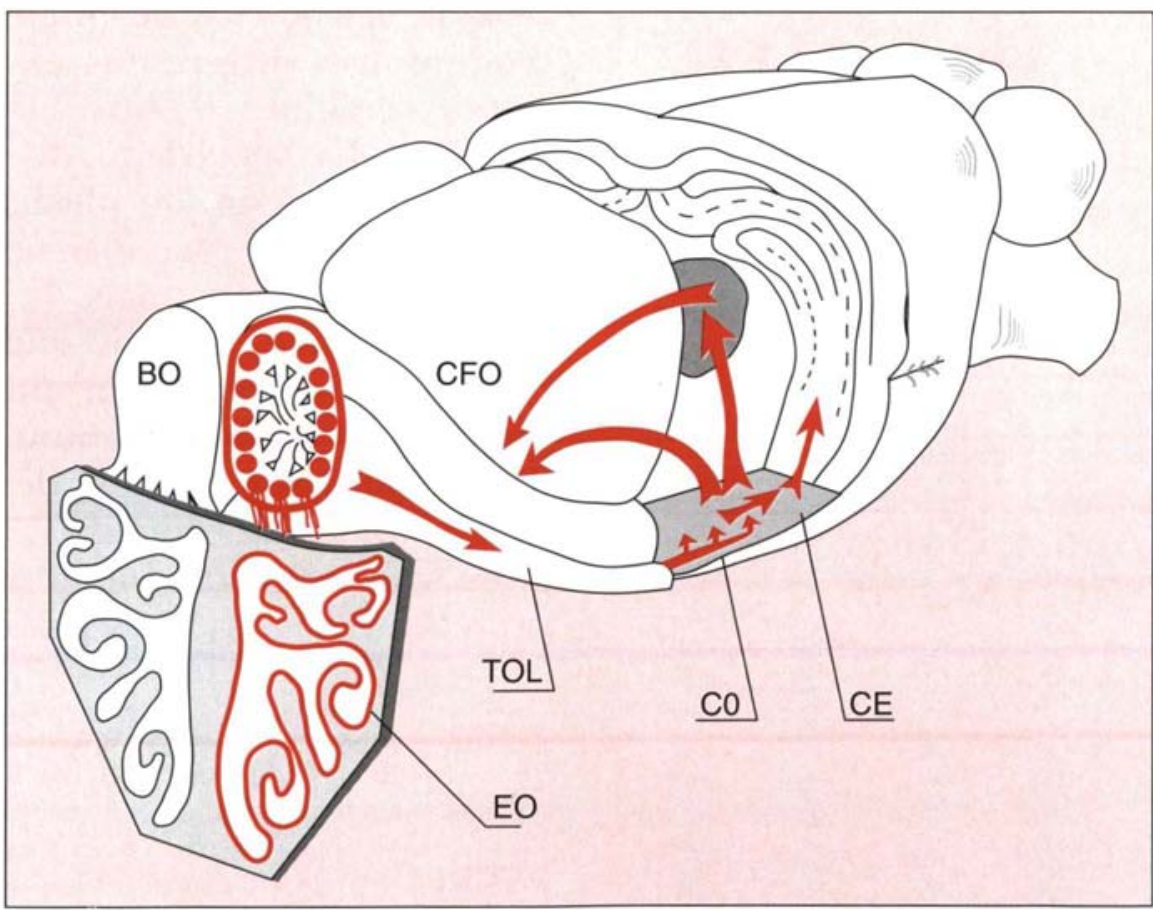

Figure 1. Représentation schématique des voies olfactives chez un mammifère. Les axones des neurorécepteurs situés dans l'épithélium olfactif (EO) rejoignent le bulbe olfactif (BO). La sortie du bulbe olfactif se projette, via le tractus olfactif latéral (TOL), sur un ensemble d'aires télencéphaliques paléocorticales groupées sous le nom de cortex olfactif (CO). De là, l'information chemine vers le néocortex fronto-orbitaire (CFO) soit par une voie directe, soit par une voie indirecte par le thalamus. Le cortex entorhinal (CE), en continuité avec le cortex olfactif est la voie d'entrée dans l'hippocampe. Des projections vers l'amydgale et l'hypothalamus (non montrées) constituent $d^{\prime}$ autres accès du système olfactif au système hypothalamo-limbique. 


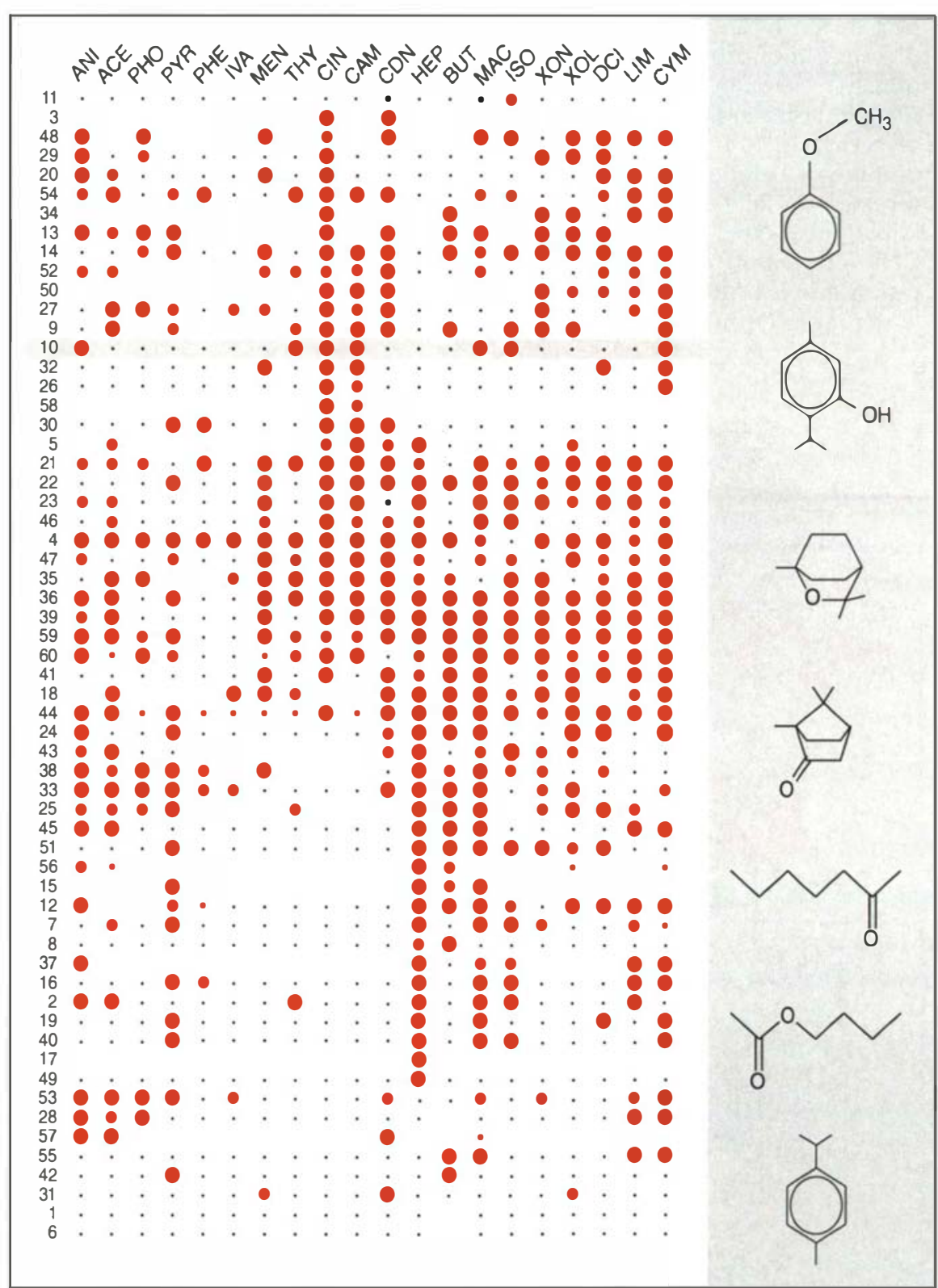

Figure 2. La sensibilité complexe des cellules réceptrices olfactives. Le tableau représente symboliquement les réponses de cellules réceptrices individuelles (lignes) stimulées par 20 odorants purs (colonnes). Le diamètre des points rouges est proportionnel à la fréquence maximum des potentiels d'action émis. Les petits points noirs correspondent à l'activité spontanée en absence de réponse. Dans la colonne grisée à droite, sont représentées les 7 molécules actives sur la cellule réceptrice sensorielle $n^{\circ} 10$. Leurs structures sont si différentes que cela suggère, soit que la stimulation a été relayée par plusieurs types de récepteurs, soit que ces derniers sont trés peu sélectifs. ( $D^{\prime}$ après une figure modifiée de [6]). cune propriété physico-chimique prise isolément, même mesurée avec la plus extrême précision, ne saurait désigner sans ambiguïté la molécule qui l'exprime parmi toutes les autres. Les récepteurs seraient-ils donc des détecteurs de propriétés physico-chimiques fondamentales? Bien que les découvertes récentes n'aient rien dit sur ce point, tout porte à croire qu'il n'en est rien et que les sites actifs des récepteurs sont configurés pour reconnaître des molécules, non des propriétés isolées.

\section{La représentation distribuée de l'odeur}

A première vue, le système olfactif apporte donc une réponse simple au problème qui se pose à lui. Ayant à détecter et reconnaître des molécules, il le fait en dotant son entrée d'un grand nombre de détecteurs moléculaires aux propriétés réceptrices diversifiées. Faut-il en conclure que la perception de toute odeur se résume à l'activation d'un récepteur particulier, à la transduction puis à la transmission du message par une voie spécifique jusqu'aux aires cérébrales où l'information est utilisée pour la perception consciente et le contrôle du comportement (figure 1)? Il y a plusieurs raisons de douter de la réalité d'un schéma aussi simple. Une raison théorique d'abord: même si le nombre de types de récepteurs est élevé, il reste, selon toute probabilité, encore très inférieur à celui des molécules odorantes effectivement discriminables qui sont des dizaines de milliers. L'équation : "un récepteur = une odeur $"$ n'est certainement pas généralisable.

Les données neurophysiologiques issues de l'étude des cellules réceptrices ou neurorécepteurs contribuent également à corriger l'image trop simple de l'équivalence récepteur-odeur. En recueillant les réponses électriques individuelles de neurorécepteurs stimulés par une large variété d'odorants, on constate que ces cellules primaires n'ont pas une sensibilité étroitement sélective $[5,6]$. Bien au contraire, elles se montrent individuellement sensibles à des molécules de structures chimiques et d'odeurs très différentes (figure 2). Pour expliquer cette observation, on dispose de cette alternative : 
ou bien les récepteurs ont une faible sélectivité, ou bien les cellules en expriment chacune plusieurs types. Les données récentes de biologie moléculaire ne permettent pas vraiment de choisir entre les deux hypothèses. Dans le système cellulaire baculovirus-Sf9, un récepteur olfactif recombinant, OR5, présente des réponses peu sélectives aux odorants [7], mais il reste à montrer que les propriétés observées in vitro sont bien celles du récepteur in vivo. Quant au nombre de récepteurs différents exprimés par une même cellule, une évaluation indirecte le fait considérer comme faible, peut-être égal à l'unité [8]. Cette évaluation découle de l'observation que les ARN d'une sous-famille génique donnée ne sont détectés, par hybridation in situ, que dans une faible fraction, un pour cent ou moins, de la population des neurorécepteurs [9]. Quoi qu'il en soit, un pas de plus dans le sens de la non sélectivité cellulaire est franchi lorsque s'effectue, dans les glomérules du bulbe olfactif, la convergence synaptique de plusieurs centaines de neurorécepteurs sur chacun des neurones de deuxième ordre, les cellules mitrales (figure 3). De fait, les cellules mitrales présentent expérimentalement les mêmes sensibilités multiples que les neurorécepteurs, même si leur pouvoir de discrimination est dans une certaine mesure accru [10, 11].

Il faut donc renoncer à voir le système olfactif comme un faisceau de

\section{RÉFÉRENCES}

10. Duchamp A. Electrophysiological res ponses of olfactory bulb neurons to odour stimuli in the frog. A comparison with re ceptor cells. Chem Senses $1982 ; 2: 191-210$.

11. Mori K, Imamura K, Mataga N. Differential specificities of single mitral cells in rabbit olfactory bulb for a homologous series of fatty acid odor molecules. J Neurophysiol $1992 ; 67: 786-9$

12. Gross-Isserof R, Lancet D. Concentration-dependent changes of perceived odor quality. Chem Senses 1988 ; 13 : 191-204.

13. Amoore JE. Specific anosmia : a clue to the olfactory code. Nature $1967 ; 214: 1095$ 8.

14. Pourtier L, Sicard G. Comparison of the sensitivity of $\mathrm{C} 57 / \mathrm{BL} / 6 \mathrm{~J}$ and AKR/J mice to airborne molecules of isovaleric acid and amyl acetate. Behav Genet 1990 ; 20 : 499501 .

15. Wissell-Buechy D, Amoore JE. Odourblindness to musk : simple recessive inheritance. Nature $1973 ; 242$ : 271-3.

16. Wysocki CJ, Beauchamp GK. Ability to smell androstenone is genetically determined. Proc Natl Acad Sci USA 1984 ; 81 : 4899 902.

17. Astic L, Saucier D, Holley A. Topographical relationships between olfactory receptor cells and glomerular foci in the rat olfactory bulb. Brain Res $1987 ; 424: 14452$

18. Vassar R, Ngai J, Axel R. Spatial segregation of odorant receptor expression in the mammalian olfactory epithelum. Cell 1993 ;

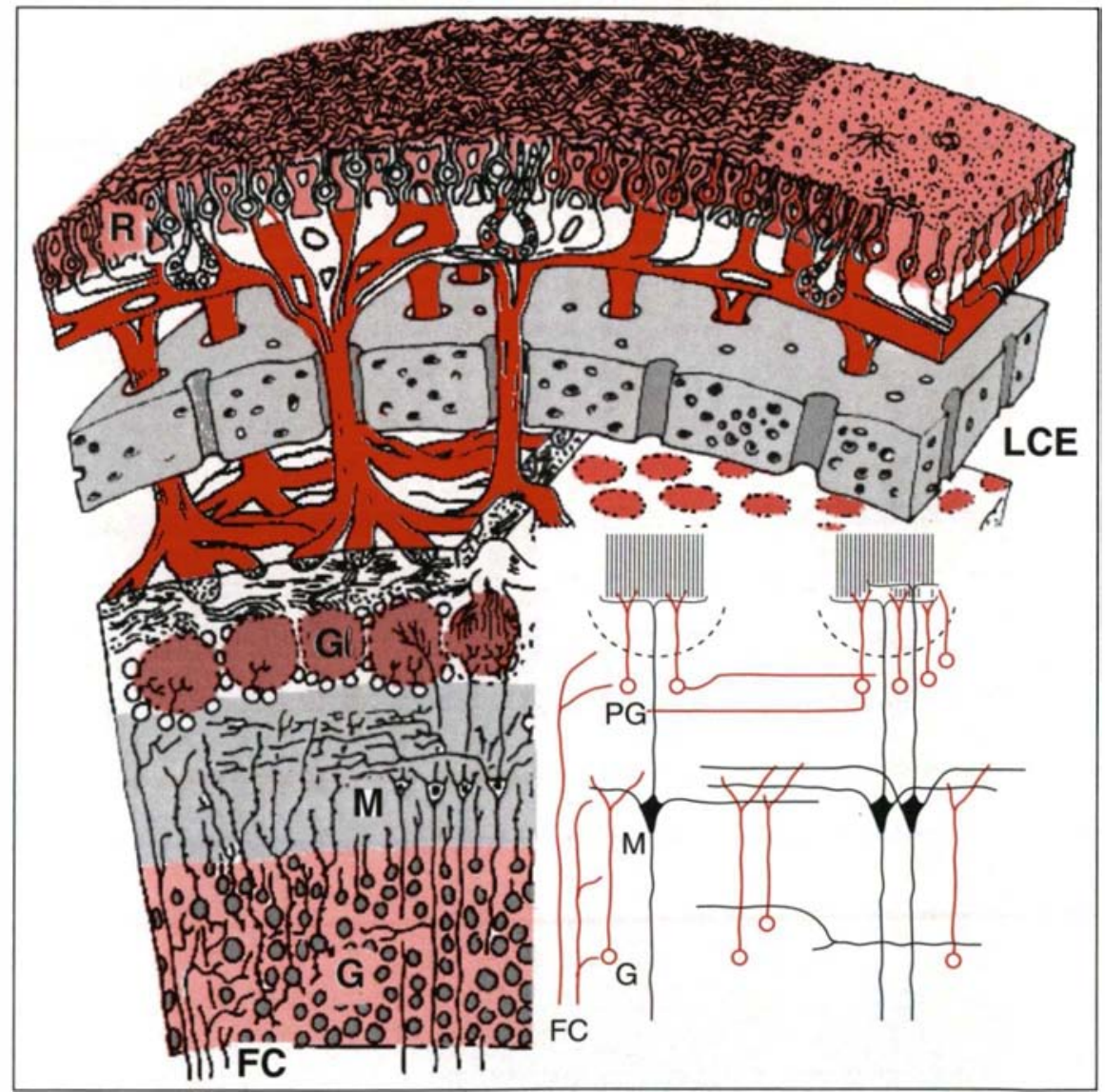

Figure 3. Relations entre l'épithélium olfactif et le bulbe olfactif et organisation interne du bulbe. Les cellules réceptrices $(R)$ de la muqueuse olfactive envoient leurs axones à l'entrée du bulbe olfactif, à travers la lame criblée de l'ethmoïde (LCE), dans les glomérules (GI) où ils font synapse avec les dendrites primaires des cellules mitrales (M). Ces neurones de projection sont contrôlés par deux catégories de neurones locaux inhibiteurs, les cellules périglomérulaires (PG) et les cellules granulaires $(G)$. Le bulbe olfactif reçoit plusieurs contingents de "fibres centrifuges" (FC) provenant du tronc cérébral, du télencéphale basal et des aires de projection des cellules mitrales. 
voies parallèles conduisant des messages parfaitement singularisés dès leur origine. Un récepteur détecte et donc confond - plusieurs odorants agonistes et, si pur que soit le stimulus, il est reconnu par plusieurs types de récepteurs et mobilise des neurones qui ne lui sont pas spécifiquement dédiés. Un neurone considéré isolément ne fournit qu'une indication ambiguë sur la nature du stimulus qui a déclenché son activité. Notons que le nombre et la nature des récepteurs impliqués ne sont pas indépendants de la concentration à laquelle le stimulus est délivré, comme en témoignent les nombreux exemples de variations de la qualité de l'odeur avec son intensité [12]. Une entité moléculaire donnée, dans une certaine plage de concentration, est donc codée sous la forme d'un motif plurineuronal d'activité au sein duquel l'information a un caractère distribué. Après avoir régressé en apparence dans les étapes intermédiaires de la transmission, la sélectivité et le pouvoir de résolution du système réapparaissent à l'issue du traitement de l'information puisque les réponses des sujets manifestent une capacité de discrimination qui est très proche du maximum théorique. En effet, seules certaines formes énantiomériques d'odorants, mais non toutes, ne peuvent être distinguées par des sujets attentifs et convenablement entraînés. Le problème qui est alors posé est celui de comprendre comment les réseaux de neurones qui reçoivent le message apparemment brouillé dont sont porteurs les neurones individuels restaurent et accroissent encore le pouvoir de résolution permis par la population diversifiée des récepteurs.

\section{Les anosmies sélectives mieux comprises}

Cette conception "populationnelle" du codage de l'odeur était explicitement ou implicitement admise avant que ne soient révélées la nature des récepteurs et leur grande diversité. Les données nouvelles n'entraînent pas une refonte complète des hypothèses sur le traitement de l'information mais invitent à les approfondir et à les affiner. Plusieurs résultats, jusqu'alors mal intégrés, peuvent également recevoir un éclairage plus sa- tisfaisant. Le point central est celui de la spécificité des récepteurs. On n'en a presque aucune connaissance directe mais les indications relatives à leur grand nombre laissent ouverte la possibilité qu'une partie d'entre eux aient un degré élevé de sélectivité. Lorsqu'on postulait un nombre limité de récepteurs, disons quelques dizaines de types seulement, on était nécessairement conduit à penser que chacun d'eux acceptait un grand nombre de ligands. Le corollaire était qu'un odorant donné devait nécessairement activer plusieurs récepteurs. On comprenait mal, dans ce cas, qu'une faible modification apportée à une molécule eût pour conséquence, comme on l'observait parfois dans des études de relation structure-activité, non seulement de changer profondément son odeur, mais également d'élever son seuil de perception de façon considérable. Il eût fallu accepter l'idée que la perception de l'odorant reposait pour l'essentiel sur l'activité d'un seul récepteur, ce qui était en contradiction avec les présupposés.

L'étude des "anosmies sélectives" soulevait le même type d'interrogation. Des sujets humains [13], ainsi que des animaux [14], ne perçoivent pas certaines familles d'odorants ou ne les détectent qu'à forte concen tration, alors qu'ils sont normalement sensibles au reste de l'univers des odeurs. Ces anomalies limitées étant génétiquement transmises [15, 16], il était tentant de postuler qu'elles avaient pour origine l'absence ou le dysfonctionnement d'un récepteur. Mais en associant un récepteur à chaque forme d'anosmie sélective rencontrée, on s'engageait dans une voie qui avait le défaut de requérir beaucoup plus de récepteurs que l'on croyait pouvoir en postuler. La richesse annoncée du répertoire de récepteurs permet désormais d'imaginer une situation diversifiée dans laquelle certains récepteurs reconnaîtraient très sélectivement un petit nombre de ligands - par exemple ceux impliqués dans les anosmies sélectives - tandis que d'autres se montreraient nettement moins sélectifs. Les odorants les plus susceptibles d'une reconnaissance sélective sont, sans conteste, les molécules de structure rigide et de poids moléculaire relativement élevé, produites par des organismes vivants et susceptibles d'avoir assumé des rôles de signaux de communication interindividuelle à quelque stade antérieur de l'évolution du groupe zoologique. Quant aux molécules plus petites ou peu rigides, elles seraient détectées par un nombre plus grand de récepteurs, au prix, sans doute, d'une moindre affinité pour chacun d'eux.

Ces remarques conduisent à voir le système olfactif périphérique comme un système hétérogène quant aux solutions adoptées pour reconnaître, au niveau moléculaire, la grande variété des stimuli qu'il reçoit. Quoi qu'il en soit, du fait de la sensibilité plurielle qui paraît être la règle générale au niveau cellulaire, au moins chez les amphibiens étudiés, l'hypothèse du codage de l'odeur en termes de motif plurineuronal d'activité conserve toute sa valeur. Il convient alors de s'interroger sur la représentation de l'odeur à l'entrée du cerveau.

\section{Les glomérules olfactifs et la chimiotopie}

Le bulbe olfactif qui reçoit le message afférent contient les neurones de deuxième ordre, cellules mitrales et cellules à panache, ainsi que les deux principales catégories d'interneurones : les cellules périglomérulaires, GABAergiques et dopaminergiques, et les cellules granulaires, (AABAergiques (figure 3). Ces éléments sont organisés en couches concentriques au sein de la structure dont la principale singularité anatomique est représentée par une couche de quelques milliers de glomérules sousjacents aux fibres du nerf olfactif. En leur sein sont concentrées les relations synaptiques d'entrée entre les axones des neurorécepteurs et les dendrites primaires de groupes de cellules mitrales. Le neurotransmetteur de ces synapses est encore inconnu. Si l'on était tenté de considérer le bulbe comme un simple relais de la voie olfactive afférente, on en serait dissuadé en considérant le grand nombre de "fibres centrifuges " qu'il reçoit en retour des aires sur lesquelles il se projette - notamment du cortex piriforme - et des régions d'origine des grands systèmes noradrénergique, sérotoninergique et cholinergique. 
La connaissance de l'organisation spatiale des afférences est une étape importante de l'étude du bulbe en tant que système de traitement d'information. Chez le rat, malgré la complexité des fosses nasales, il est possible d'établir une correspondance assez précise entre des zones du neuroépithélium et des territoirescibles du bulbe olfactif, territoires allongés selon l'axe antéro-postérieur [17] (figure 4). Cette projection, régiono-régionale plutôt que vraiment somatotopique, autorise des convergences et des divergences à l'intérieur des aires en correspondance.

Cette organisation anatomique soustend-elle une organisation fonctionnelle ? On sait depuis longtemps que toutes les régions de l'épithélium olfactif ne sont pas également sensibles à tous les odorants. L'existence d'une chimiotopie vient de trouver une confirmation dans des expériences d'hybridation in situ [9, 18]. Elles montrent que des neurones sensoriels exprimant un récepteur donné sont localisés dans une aire limitée de l'épithélium olfactif où ils représentent alors environ un pour cent de la population cellulaire. La notion de chimiotopie s'applique également au bulbe olfactif. En effet, lors de la stimulation par une odeur, les différents glomérules incorporent inégalement le 2-désoxyglucose radioactif, marqueur de l'activité métabolique neuronale [19]. Chaque

\section{RÉFÉRENCES}

19. Jourdan F, Duveau A, Astic L, Holley A. Spatial distribution of (14C)2-deoxyglucose uptake in the olfactory bulbs of rat stimulated with two different odours. Brain Res $1980 ; 188: 139-54$.

20. Mori K. Molecular and cellular properties of mammalian primary olfactory axons. Microscop Res Tech 1993 ; 24 : 131-41.

21. Buonviso N, Chaput M, Berthommier F. Temporal patterns analyses in pairs of neighboring mitral cells. I Neurophysiol 1992; 68: 417-24.

22. Le Jeune $H$, Jourdan $F$. Cholinergic innervation of olfactory glomeruli in the rat : an ultrastructural immunocytochemical study. J Comp Neurol 1993 ; 336 : 279-92.

23. Freeman W. La physiologie de la perception. Pour La Science 1991 ; 162 : 70-8.

24. Gervais R, Holley A, Keverne EB. The importance of central noradrenergic influences on the rat olfactory bulb in the processing of learned olfactory cues. Chem penses $1988 ; 13: 3-12$.

25. Gray CM, Freeman WJ, Skinner JE. Chemical dependencies of learning in the rabbit olfactory bulb : acquisition of the transient spatial pattern change depends on norepinephrine. Behav Neurosc $1986 ; 100$, $4: 585-96$.

26. Ravel N, Elaagouby A, Gervais R. Scopolamin injection into the olfactory bulb impairs short-term olfactory memory in rats. Behav Neurose 1994 ; 108 : 317-24

27. Mouly AM, Kindermann U, Gervais $R$, Holley $A$. Involvement of the olfactory bulb in consolidation processes associated with long-term memory in tats. Behav Neurose $1993 ; 107: 451-7$.

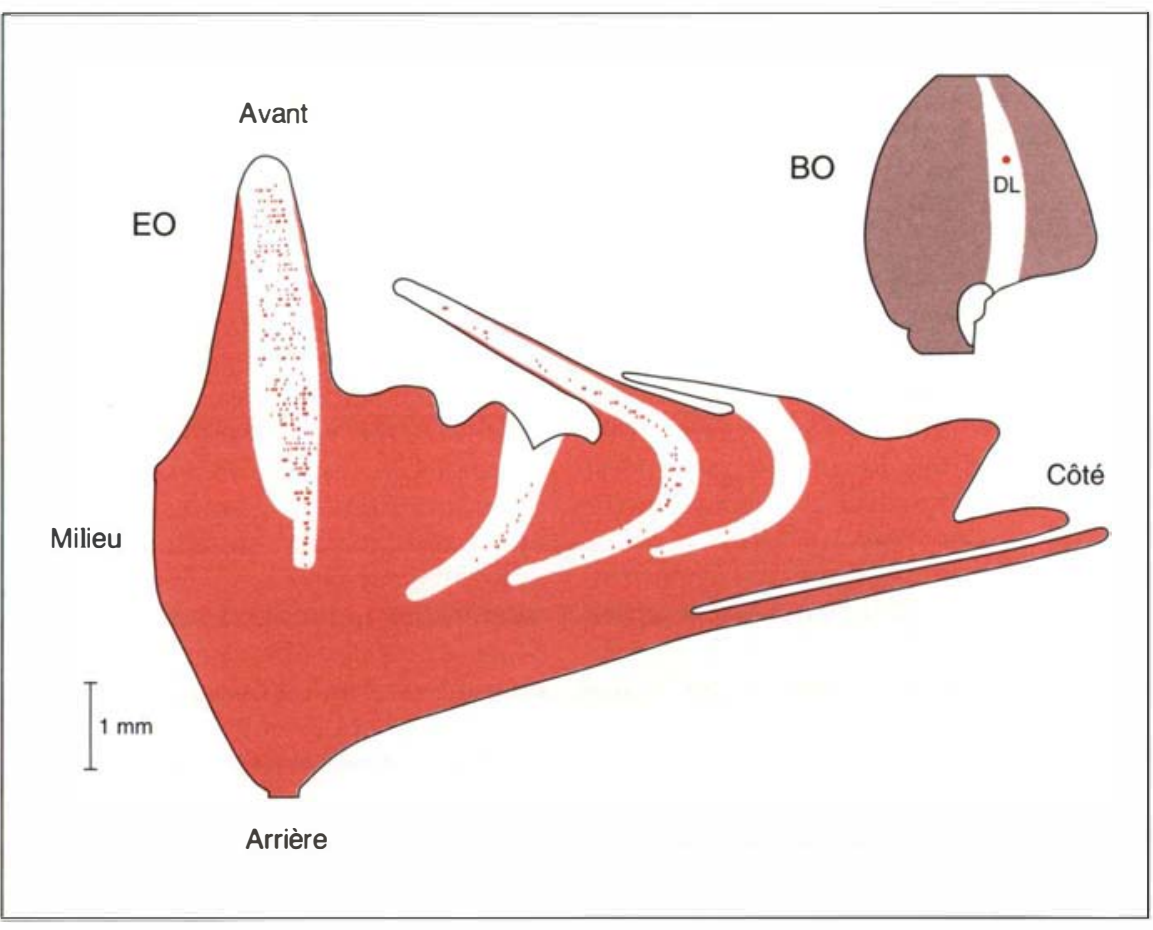

Figure 4. Projections des neurorécepteurs sur le bulbe olfactif. L'épithélium olfactif et le bulbe olfactif d'un rat sont représentés comme si leur surface était déroulée. Une micro-injection de peroxydase du raifort dans un glomérule du bulbe olfactif (BO) (point dans le secteur dorso-latéral, DL) produit le marquage rétrograde de neurorécepteurs confinés dans quelques territoires circonscrits de l'épithélium (EO) (points dans les zones claires). Des injections pratiquées dans des glomérules alignés dans la direction antéro-postérieure du même secteur bulbaire marquent des neurorécepteurs très majoritairement situés dans les mêmes territoires de l'épithélium (non montré). $C^{\prime}$ est la notion de projection régiono-régionale. Dans cette représentation, la dimension antéro-postérieure des deux structures est dilatée d'un facteur 3 par rapport à la dimension médio-latérale. (Modifié d'après [17]). 
odeur est ainsi représentée, à l'entrée du bulbe olfactif, par une carte d'activité fonctionnelle qui lui est propre.

La question se pose alors de savoir si la nature de la chimiosensibilité des cellules réceptrices détermine de quelque manière le regroupement de leurs axones dans les glomérules. Plusieurs types d'études éclairent cette question sans toutefois lui apporter une réponse nette. On sait tout d'abord que les axones récepteurs qui réagissent semblablement à des marqueurs immunologiques de molécules de surface convergent dans les mêmes ensembles de glomérules et ne se mêlent pas aux axones qui présentent une réactivité différente [20]. Par ailleurs, le marquage des glomérules par incorporation de 2désoxyglucose sous stimulation par un odorant pur offre un caractère contrasté [19] qui pourrait être ex- pliqué par un fort taux de convergence de neurorécepteurs de même sensibilité dans un sous-ensemble restreint de glomérules. On a parlé à ce propos de l'unité fonctionnelle des glomérules. Des travaux d'électrophysiologie [21] ont introduit une notion un peu différente : celle du fonctionnement unitaire du glomérule. Par l'enregistrement simultané de l'activité de paires de cellules mitrales supposées innerver un même glomérule on a montré une forte tendance de ces cellules appariées à répondre semblablement aux mêmes stimulus, alors que des paires de cellules innervant des glomérules adjacents tendaient à montrer des réponses de signes opposés (excitation contre inhibition). Cette dernière observation doit être mise au compte de l'inhibition interglomérulaire relayée par les cellules périglomérulaires. L'organisation glomérulaire

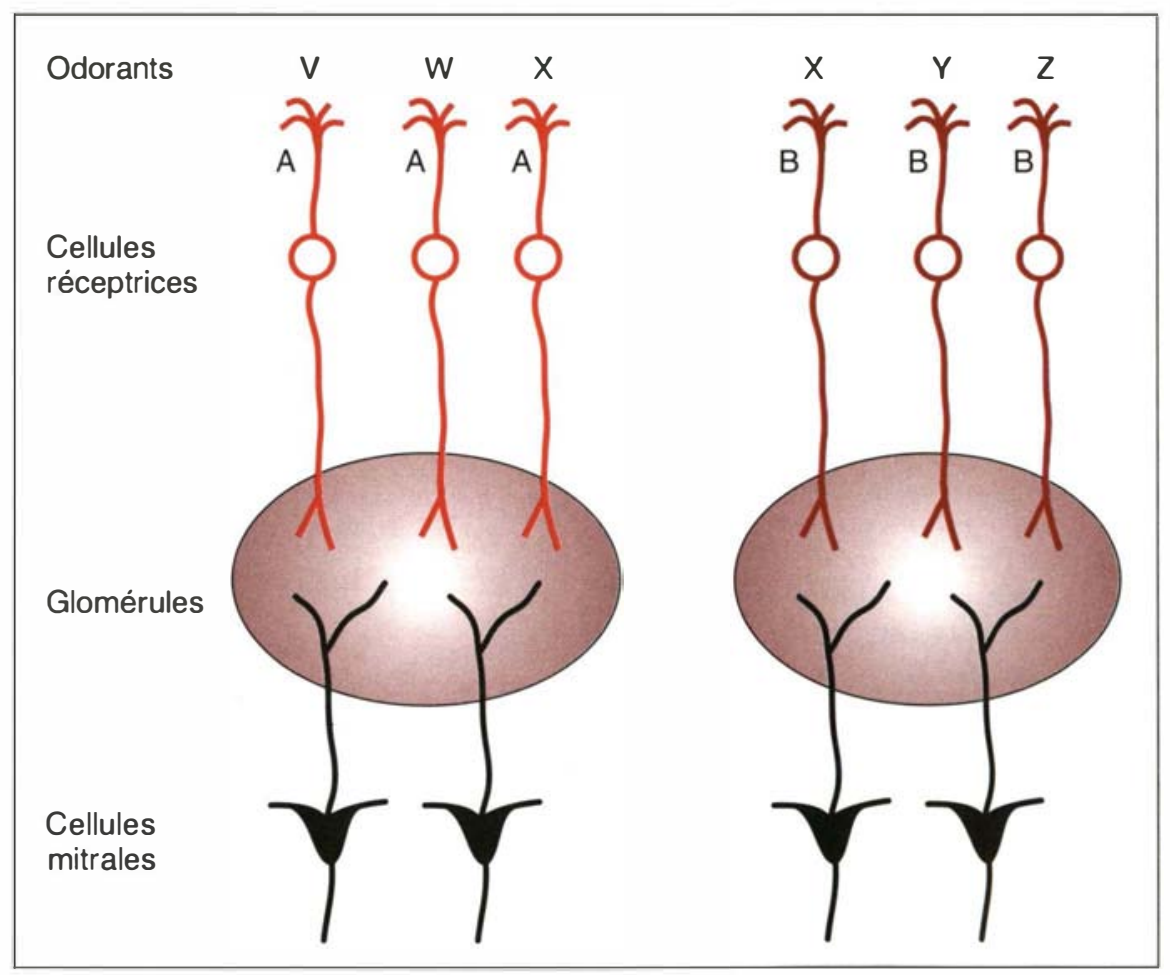

Figure 5. Schématisation d'une hypothèse actuelle sur l'organisation des projections des cellules réceptrices olfactives. Selon cette hypothèse, les cellules réceptrices exprimant un même récepteur (ici $A$ ou $B$ ) regrouperaient leurs axones dans les mêmes glomérules et donc communiqueraient leur excitation aux populations distinctes de cellules mitrales innervant ces glomérules. Cette relation récepteur-glomérule n'est pas équivalente à une relation odeur-glomérule puisque les récepteurs sont supposés non étroitement sélectifs. Dans le cas représenté l'odorant $x$ activera les deux glomérules. L'hy pothèse suppose que les cellules n'expriment chacune qu'un seul récepteur, ce qui demande confirmation. pourrait donc avoir pour conséquence, selon un mécanisme qui reste non élucidé - similarité des entrées, distribution synaptique homogène de ces entrées, diffusion intraglomérulaire du transmetteur... - de conférer le même profil de chimiosensibilité aux quelques dizaines de cellules mitrales inféodées à un même glomérule. En termes de communication cellulaire la conséquence en serait une réduction considérable du nombre de canaux de transfert d'informations, puisque ce nombre serait équivalent à celui des glomérules et non à celui des cellules mitrales.

L'hypothèse la plus couramment avancée depuis la découverte de la variété des récepteurs est celle d'une convergence, vers les mêmes glomérules, des cellules sensorielles exprimant le même récepteur (figure 5). Ce n'est qu'une hypothèse. Sans nécessairement supposer une correspondance stricte, bi-univoque entre récepteurs moléculaires et glomérules, on peut postuler une certaine spécialisation de ces glomérules et, partant, des groupes de cellules mitrales qui les innervent. Sans trancher ce débat, des données morphologiques et immunohistochimiques confirment que tous les glomérules ne sont pas équivalents puisque certains d'entre eux, qualifiés d'" atypiques ", sont distingués par la richesse exceptionnelle de leur innervation cholinergique [22].

\section{L'intégration olfacto-bulbaire et sa plasticité}

A chaque inhalation, une carte d'activité s'inscrit à l'entrée du bulbe olfactif et vient interagir avec l'importante activité spontanée des neurones bulbaires. Cette carte, d'abord glomérulaire, est traitée par les interneurones périglomérulaires qui en accusent les contrastes en transmettant une inhibition de voisinage et progresse vers des couches plus profondes via les dendrites primaires des cellules mitrales. Lorsque l'excitation atteint l'importante population de cellules granulaires GABAergiques, l'inhibition en retour des cellules mitrales intervient et amorce une oscillation cohérente de l'ensemble du bulbe, donnant sur l'électroencéphalogramme un motif d'ondes modu- 
lées spatialement dans leur amplitude et leur phase [23]. Les ondes extracellulaires n'étant que le reflet des variations du potentiel membranaire des neurones, elles révèlent les fluctuations concomitantes de l'excitabilité des cellules mitrales et de la probabilité d'émission de leurs influx. La carte d'activité imposée à la couche glomérulaire d'entrée donne ainsi naissance à un motif plurineuronal de sortie, dirigé vers les aires paléocorticales, motif qui incorpore également dans sa structure spatiofréquentielle des caractéristiques déterminées par l'état des circuits neuronaux bulbaires.

Les recherches actuelles s'efforcent d'évaluer le degré de plasticité de ces circuits, de déterminer les facteurs qui influent sur leur fonctionnement et d'en interpréter les conséquences sur le traitement de l'information. C'est ainsi que l'attention est attirée vers les fonctions des fibres efférentes, dites "centrifuges", qui font synapse avec les interneurones locaux, cellules périglomérulaires et granulaires. Plusieurs travaux impliquent, par exemple, les fibres noradrénergiques dans des formes de plasticité sous-tendant des apprentissages olfactifs [24]. Il semble que soient spécifiquement renforcés, par libération de noradrénaline, des motifs d'activité codant des odeurs reçues dans certaines conditions d'apprentissage [25]. D'autres travaux montrent que la mémoire à court terme de l'odeur est détériorée lorsque le fonctionnement du système cholinergique du bulbe est localement perturbé [26]. Quant à l'apprentissage à long terme, sa consolidation échoue si l'activité intrinsèque du bulbe est momentanément réduite par micro-injection locale d'un anesthésique dans un délai de quelques dizaines de minutes suivant l'acquisition [27].

L.es recherches sur le système olfactif ont jusqu'à maintenant progressé à la périphérie des grands chantiers des neurosciences. Bien des voies de recherche originales ont cependant été ouvertes. Déjà, la découverte des récepteurs olfactifs entraîne l'engagement de nouveaux chercheurs et l'introduction de nouvelles méthodes. Il faudra sans doute beaucoup de temps pour dévoiler avec toute la tion des sites récepteurs protéiques et la structure fine des messages engendrés par les odeurs dans l'épithélium sensoriel. Mais l'impulsion décisive est donnée

\section{Summary}

Olfactory receptors and neural coding of odors

Current views on the neural coding of olfactory information may have to be reconsidered in the light of the discovery of a huge family of genes coding for olf actory receptors. The extreme diversity of these receptors confers a striking singularity to the olfactory system in that it apparently provides the sensory epithelium itself with a high capacity for stimulus recognition. The large number of receptors makes it possible that some odorants be predominantly recognized by receptors of a single type, which offers a basis for understanding specific anosmias. However, individual molecular sensitivities are mixed at the receptor cell level. It follows that the current concept of odor coding in terms of across-neuron pattern of activity remains a valid assumption. Odor coding patterns define a chemotopy at both epithelium and bulb levels. Several data from 2-deoxyglucose metabolic studies, immunohistology and electrophysiology suggest that olf actory glomeruli act as convergence centers for related inputs. Receiving various efferent inputs from noradrenergic, serotoninergic and cholinergic projection systems along with feedback innervation from its paleocortical projection areas, the olfactory bulb plays a key role in olf actory processing. Its direct involvement in olfactory learning and memory is a promising line of research. All these domains will benefit from a better understanding of the principles of molecular recognition in olfactory receptor cells.

\section{TIRÉS A PART}

\author{
A. Holley.
}

